\title{
GW23-e0568 ONE-YEAR FOLLOW-UP OF BLEEDING ON ANTI- PLATELET DRUG USE IN PATIENTS WITH ACUTE MYOCARDIAL INFARCTION
}

doi:10.1136/heartjnl-2012-302920k.48

${ }^{1}$ Zhou Aiqin, 'Wang Xiaoping, 'iao Wei, 'Z Zhou Aiqin. 'Department of Cardiology, The First Affiliated Hospital of Gannan University, Ganzhou 341000, China; ${ }^{2}$ The First Affiliated Hospital of Gannan University, Ganzhou 341000, China

Objectives The use of multiple antithrombotic drugs has increased the risk of major bleeding in acute myocardial infarction (AMI). We aimed to investigate the anti-platelet drug use after discharge in patients with in-hospital major bleeding.

Methods 198 patients with AMI were divided into two groups according to bleeding in hospital: bleeding group $(n=36)$ or nonbleeding group $(n=198)$. The utilisation rates of the anti-platelet drug (aspirin and clopidogrel) were collected at discharge, in the first, sixth and twelfth month out of hospital.

Results No significant differences were found in gender, age, weight, hypertension, diabetes mellitus, the history of PCI $(16.9 \%$ vs $13.6 \%, p>0.05)$ or stenting $(66.7 \%$ vs $65.2 \%, p>0.05)$. Compared with non-bleeding group, the patients in bleeding group were more older $(62.6 \pm 16.2$ vs $55.9 \pm 15.2$ year, $p<0.05)$, more with hypertension $(69.4 \%$ vs $53.0 \%, \mathrm{p}<0.05)$, more with chronic renal insufficiency $(25.0 \%$ vs $12.1 \%, p<0.05)$. The aspirin usage in bleeding group was less than that in non-bleeding group at discharge $(69.4 \%$ vs $91.9 \%, p<0.01$, in the first month $(65.7 \%$ vs $85.9 \%$, $\mathrm{p}<0.01)$ and sixth were lower $(61.6 \%$ vs $83.8 \% \mathrm{p}<0.01)$, but no 


\section{ABSTRACTS}

difference in the twelfth month $(72.2 \%$ vs $82.8 \%, \mathrm{p}>0.05)$. The clopidogrel usage in bleeding group was less than that in nonbleeding group at discharge $(66.7 \%$ vs $87.4 \% \mathrm{p}<0.01)$ and in the first month $(65.7 \%$ vs $85.9 \% \mathrm{p}<0.01)$, but no difference in the sixth $(25.0 \%$ vs $39.4 \% \mathrm{p}<0.01)$ and twelfth month $(23.5 \%$ vs $28.3 \% \mathrm{p}<0.01$ ).

Conclusions AMI patients with in-hospital major bleeding have significantly decreased 1-year utilisation rates of aspirin and clopidogrel. Further investigation is warranted to understand the prognosis underlying this relationship. 